CRYSTALLOGRAPHIC COMMUNICATIONS

ISSN 2056-9890

Received 3 January 2016

Accepted 17 January 2016

Edited by $\mathrm{H}$. Stoeckli-Evans, University of Neuchâtel, Switzerland

† Additional correspondence author, e-mail: elemam5@hotmail.com.

Keywords: crystal structure; 1,3,4-oxadiazole; thiophene; piperazine; disorder; hydrogen bonding

CCDC reference: 1447823

Supporting information: this article has supporting information at journals.iucr.org/e

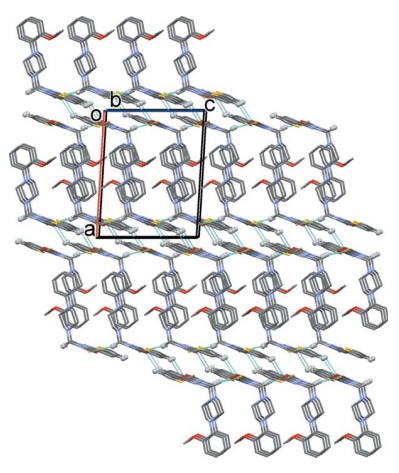

OPEN $\odot$ ACCESS

\section{Crystal structure of 3-\{[4-(2-methoxyphenyl)- piperazin-1-yl]methyl\}-5-(thiophen-2-yl)-1,3,4-oxa- diazole-2(3H)-thione}

\author{
Monirah A. Al-Alshaikh, ${ }^{a}$ Hatem A. Abuelizz, ${ }^{b}$ Ali A. El-Emam, ${ }^{b} \ddagger$ Mohammed S. M. \\ Abdelbaky ${ }^{\mathrm{C}}$ and Santiago Garcia-Granda ${ }^{\mathrm{C} *}$
}

${ }^{\mathbf{a}}$ Department of Chemistry, College of Sciences, King Saud University, Riyadh 11451, Saudi Arabia, ${ }^{\mathbf{b}}$ Department of Pharmaceutical Chemistry, College of Pharmacy, King Saud University, Riyadh 11451, Saudi Arabia, and ${ }^{\mathbf{c}}$ Department of Physical and Analytical Chemistry, Faculty of Chemistry, Oviedo University-CINN, Oviedo 33006, Spain.

*Correspondence e-mail: sgg@uniovi,es

The title compound, $\mathrm{C}_{18} \mathrm{H}_{20} \mathrm{~N}_{4} \mathrm{O}_{2} \mathrm{~S}_{2}$, is a new 1,3,4-oxadiazole and a key pharmacophore of several biologically active agents. It is composed of a methyl(thiophen-2-yl)-1,3,4-oxadiazole-2(3H)-thione moiety linked to a 2-methoxyphenyl unit via a piperazine ring that has a chair conformation. The thiophene ring mean plane lies almost in the plane of the oxadiazole ring, with a dihedral angle of $4.35(9)^{\circ}$. The 2-methoxyphenyl ring is almost normal to the oxadiazole ring, with a dihedral angle of $84.17(10)^{\circ}$. In the crystal, molecules are linked by weak $\mathrm{C}-\mathrm{H} \cdots \mathrm{S}$ hydrogen bonds and $\mathrm{C}-\mathrm{H} \cdots \pi$ interactions, forming layers parallel to the $b c$ plane. The layers are linked via weak $\mathrm{C}-\mathrm{H} \cdots \mathrm{O}$ hydrogen bonds and slipped parallel $\pi-\pi$ interactions [intercentroid distance $=$ 3.6729 (10) ̊] , forming a three-dimensional structure. The thiophene ring has an approximate $180^{\circ}$ rotational disorder about the bridging $\mathrm{C}-\mathrm{C}$ bond.

\section{Chemical context}

1,3,4-Oxadiazole derivatives are structural motifs of particular value in material sciences (Zhang et al., 2011) and agrochemistry (Shi et al., 2001; Milinkevich et al., 2009; Li et al., 2014). In addition, they occupy a unique situation in the field of medicinal chemistry as pharmacophores possessing diverse pharmacological activities including antibacterial (Ogata et al., 1971; Rane et al., 2012; Al-Omar, 2010), anticancer (Pinna et al., 2009; Gamal El-Din et al., 2015; Zhang et al., 2014; Du et al., 2013), antiviral (Summa et al., 2008; Wu et al., 2015; El-Emam et al., 2004), antihypertensive (Vardan et al., 1983; Schlecker \& Thieme, 1988), anti-inflammatory (Bansal et al., 2014; Kadi et $a l ., 2007)$ and anti-oxidant (Ma et al., 2013) activities. In continuation to our previous studies on 1,3,4-oxadiazoles (ElEmam et al., 2012), we report herein on the synthesis and crystal structure of the title compound.

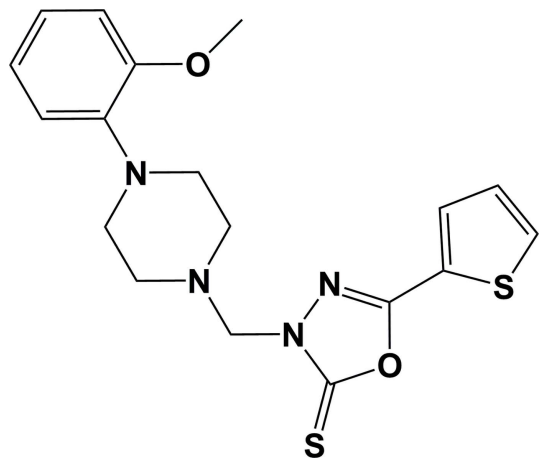




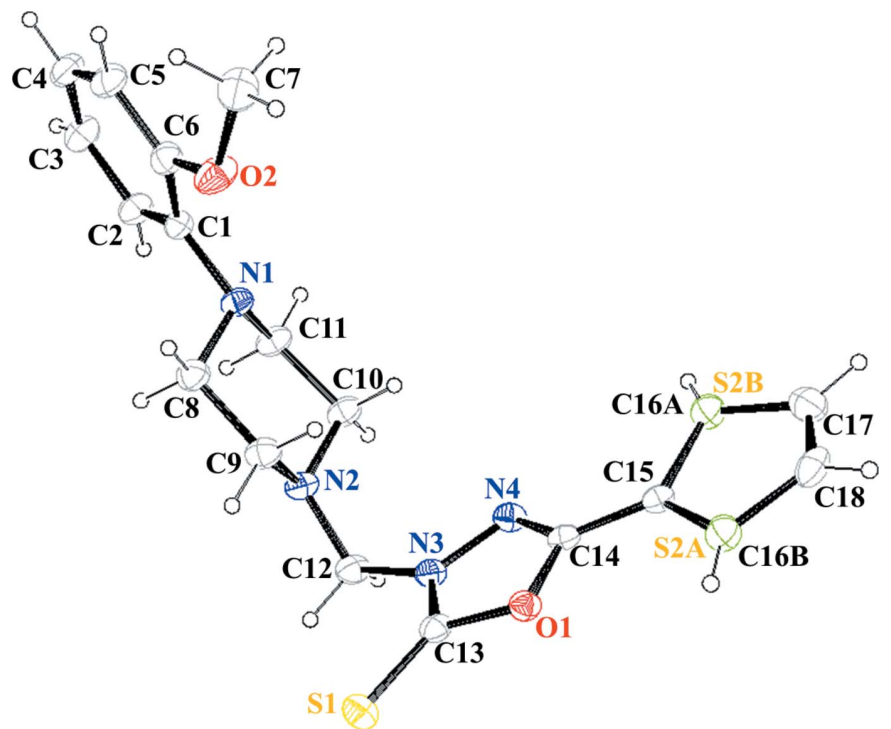

Figure 1

The molecular structure of the title compound, showing the atomlabelling scheme and displacement ellipsoids at the $50 \%$ probability level. The thiophene ring has an approximate $180^{\circ}$ rotational disorder about the bridging $\mathrm{C}-\mathrm{C}$ bond.

\section{Structural commentary}

The title compound, Fig. 1, is composed of a methyl(thiophen2-yl)-1,3,4-oxadiazole-2(3H)-thione moiety linked to a 2-methoxyphenyl unit via a bridging piperazine ring. The molecule is $\mathrm{V}$-shaped with the mean plane of the piperazine ring, that has a chair conformation, making dihedral angles of $51.2(1)$ and $77.8(1)^{\circ}$ with the 2-methoxyphenyl ring and the oxadiazole ring, respectively. The thiophene ring mean plane lies almost in the plane of the oxadiazole ring, with a dihedral angle of $4.35(9)^{\circ}$. The thiophene ring has an approximate $180^{\circ}$ rotational disorder about the bridging $\mathrm{C} 14-\mathrm{C} 15$ bond.

\section{Supramolecular features}

In the crystal, molecules are linked by weak $\mathrm{C}-\mathrm{H} \cdots \mathrm{S}$ hydrogen bonds and $\mathrm{C}-\mathrm{H} \cdots \pi$ interactions, forming layers in the $b c$ plane (Table 1 and Fig. 2). The layers are linked via $\mathrm{C}-\mathrm{H} \cdots \mathrm{O}$ hydrogen bonds and slipped parallel $\pi-\pi$ interactions $\left[C g 3 \cdots C g 1^{\mathrm{i}}=3.6729(10) \AA\right.$, inter-planar distance $=$ 3.4757 (7) $\AA$, slippage $=0.967 \AA ; C g 1$ and $C g 3$ are the centroids of the $\mathrm{S} 2 A / \mathrm{C} 15 / \mathrm{C} 16 A / \mathrm{C} 17 / \mathrm{C} 18$ and $\mathrm{O} 1 / \mathrm{N} 3 / \mathrm{N} 4 / \mathrm{C} 13 /$ C14 rings, respectively; symmetry code (i): $-x+2,-y+1$, $-z+2$ ], forming a three-dimensional structure (Table 1 and Fig. 2).

\section{Database survey}

A search of the Cambridge Structural Database (Version 5.37, last update November 2015; Groom \& Allen, 2014) for the 3-methyl-5-(thiophen-2-yl)-1,3,4-oxadiazole-2(3H)-thione moiety of the title compound gave three hits. Two of these compounds also contain a substituted piperazine ring, namely
Table 1

Hydrogen-bond geometry $\left(\AA,^{\circ}\right)$.

$C g 1$ is the centroid of the $\mathrm{S} 2 A / \mathrm{C} 15 / \mathrm{C} 16 A / \mathrm{C} 17 / \mathrm{C} 18$ ring.

\begin{tabular}{lllll}
\hline$D-\mathrm{H} \cdots A$ & $D-\mathrm{H}$ & $\mathrm{H} \cdots A$ & $D \cdots A$ & $D-\mathrm{H} \cdots A$ \\
\hline $\mathrm{C} 12-\mathrm{H} 12 A \cdots \mathrm{S} 1^{\mathrm{i}}$ & 0.97 & 2.95 & $3.860(2)$ & 157 \\
$\mathrm{C} 17-\mathrm{H} 17 \cdots \mathrm{O} 1^{\text {ii }}$ & 0.93 & 2.69 & $3.475(2)$ & 143 \\
$\mathrm{C} 5-\mathrm{H} 5 \cdots C g 1^{\text {iii }}$ & 0.93 & 2.95 & $3.660(2)$ & 135 \\
\hline
\end{tabular}

Symmetry codes: (i) $\quad x,-y+\frac{1}{2}, z-\frac{1}{2} ; \quad$ (ii) $\quad-x+2, y+\frac{1}{2},-z+\frac{5}{2}$;

3-[(4-phenylpiperazin-1-yl)methyl]-5-(2-thienyl)-1,3,4-oxadiazole-2(3H)-thione (IDOBUA; El-Emam et al., 2013) and 3-[(4-benzylpiperazin-1-yl)methyl]-5-(thiophen-2-yl)-2,3-dihydro-1,3,4-oxadiazole-2-thione (VUBYUO; Al-Omary et al., 2015). In both of these molecules, the conformation is very similar to that of the title compound.

\section{Synthesis and crystallization}

To a solution of 5-(thiophen-2-yl)-1,3,4-oxadiazole-2-thiol (920 mg, $5 \mathrm{mmol})$, in ethanol (15 ml), 1-(2-methoxyphenyl)piperazine (960 mg, $5 \mathrm{mmol}$ ) and 37\% formaldehyde solution $(1.0 \mathrm{ml})$ were added and the mixture was stirred at room temperature for $3 \mathrm{~h}$ and then allowed to stand overnight at

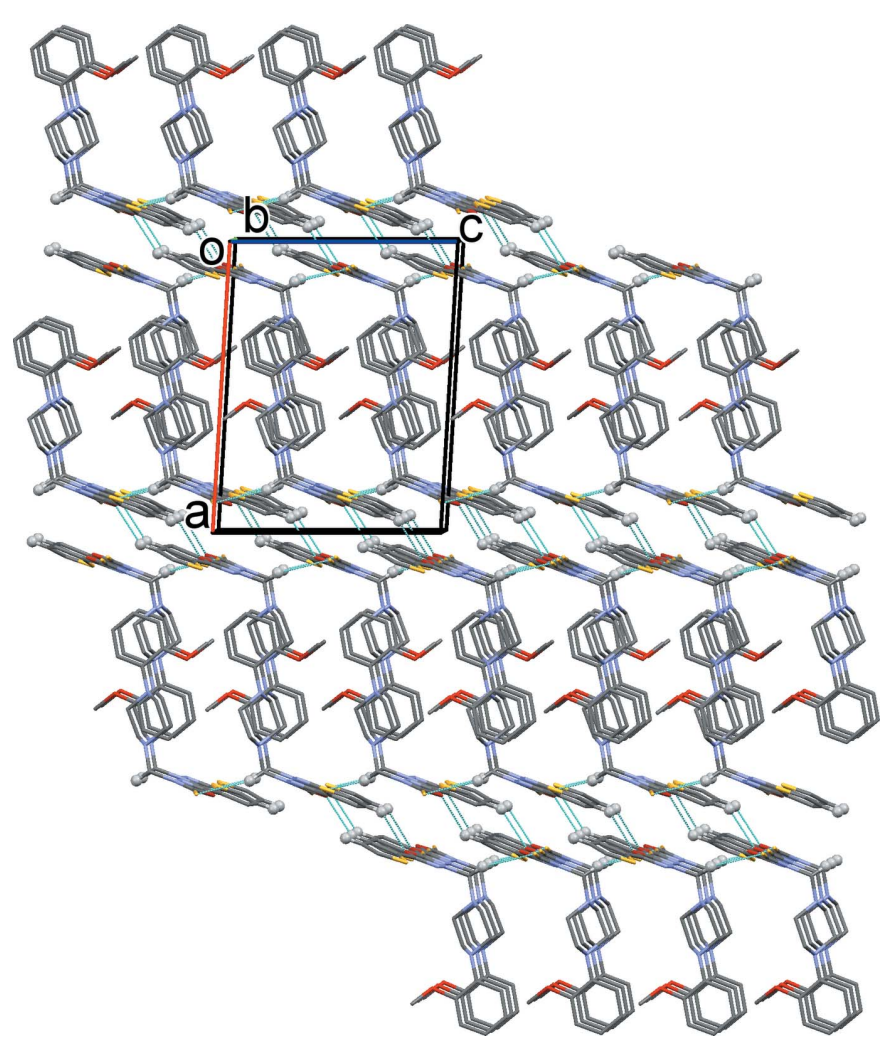

Figure 2

Crystal packing of the title compound, viewed along the $b$ axis, showing the $\mathrm{C}-\mathrm{H} \cdots \mathrm{S}$ and $\mathrm{C}-\mathrm{H} \cdots \mathrm{O}$ hydrogen bonds (Table 1 ) as dashed lines. Only $\mathrm{H}$ atoms involved in intermolecular interactions have been included. 
Table 2

Experimental details.

\begin{tabular}{|c|c|}
\hline \multicolumn{2}{|l|}{ Crystal data } \\
\hline Chemical formula & $\mathrm{C}_{18} \mathrm{H}_{20} \mathrm{~N}_{4} \mathrm{O}_{2} \mathrm{~S}_{2}$ \\
\hline$M_{\mathrm{r}}$ & 388.5 \\
\hline Crystal system, space group & Monoclinic, $P 2_{1} / c$ \\
\hline Temperature $(\mathrm{K})$ & 100 \\
\hline$a, b, c(\AA)$ & $\begin{array}{l}15.2925(2), 10.0745(1), \\
\quad 11.9726(1)\end{array}$ \\
\hline$\beta\left(^{\circ}\right)$ & $93.413(1)$ \\
\hline$V\left(\AA^{3}\right)$ & $1841.28(3)$ \\
\hline$Z$ & 4 \\
\hline Radiation type & $\mathrm{Cu} K \alpha$ \\
\hline$\mu\left(\mathrm{mm}^{-1}\right)$ & 2.80 \\
\hline Crystal size $(\mathrm{mm})$ & $0.70 \times 0.51 \times 0.41$ \\
\hline \multicolumn{2}{|l|}{ Data collection } \\
\hline Diffractometer & Agilent Xcalibur Ruby Gemini \\
\hline Absorption correction & $\begin{array}{l}\text { Multi-scan (CrysAlis PRO; } \\
\text { Agilent, 2014) }\end{array}$ \\
\hline$T_{\min }, T_{\max }$ & $0.225,0.315$ \\
\hline $\begin{array}{l}\text { No. of measured, independent and } \\
\text { observed }[I>2 \sigma(I)] \text { reflections }\end{array}$ & $13494,3545,3401$ \\
\hline$R_{\text {int }}$ & 0.026 \\
\hline$(\sin \theta / \lambda)_{\max }\left(\AA^{-1}\right)$ & 0.612 \\
\hline \multicolumn{2}{|l|}{ Refinement } \\
\hline$R\left[F^{2}>2 \sigma\left(F^{2}\right)\right], w R\left(F^{2}\right), S$ & $0.045,0.113,1.04$ \\
\hline No. of reflections & 3494 \\
\hline No. of parameters & 230 \\
\hline $\mathrm{H}$-atom treatment & $\mathrm{H}$-atom parameters constrained \\
\hline$\Delta \rho_{\max }, \Delta \rho_{\min }\left(\mathrm{e} \AA^{-3}\right)$ & $0.95,-0.65$ \\
\hline
\end{tabular}

Computer programs: CrysAlis CCD and CrysAlis RED (Oxford Diffraction, 2006), SHELXS97 and SHELXL97 (Sheldrick, 2008), ORTEP-3 for Windows and WinGX (Farrugia, 2012), Mercury (Macrae et al., 2008) and publCIF (Westrip, 2010).

room temperature. The precipitated crude product was filtered, washed with cold ethanol, dried, and crystallized from ethanol to yield the title compound as pale-yellow prismatic crystals(yield $1.67 \mathrm{~g}, 86 \%$; m.p. 419-421 K). Single crystals suitable for $\mathrm{X}$-ray analysis were obtained by slow evaporation of a $\mathrm{CHCl}_{3}:$ EtOH solution $(1: 1 ; 15 \mathrm{ml})$ at room temperature. ${ }^{1} \mathrm{H}$ NMR $\left(\mathrm{CDCl}_{3}, 500.13 \mathrm{MHz}\right): \delta 3.10(s, 8 \mathrm{H}$, piperazine-H), $3.85\left(s, 3 \mathrm{H}, \mathrm{OCH}_{3}\right), 5.15(s, 2 \mathrm{H}, \mathrm{CH} 2), 6.85-6.87(m, 1 \mathrm{H}, \mathrm{Ar}-$ $\mathrm{H}), 6.92-6.95$ ( $m, 2 \mathrm{H}, \mathrm{Ar}-\mathrm{H}), 7.01-7.03$ ( $m, 1 \mathrm{H}, \mathrm{Ar}-\mathrm{H}), 7.18(t$, $1 \mathrm{H}$, thiophene-H, $J=4.5 \mathrm{~Hz}), 7.59(\mathrm{~d}, 1 \mathrm{H}$, thiophene-H, $J=$ $4.5 \mathrm{~Hz}), 7.75(d, 1 \mathrm{H}$, thiophene-H, $J=4.5 \mathrm{~Hz}) .{ }^{13} \mathrm{C} \mathrm{NMR}$ $\left(\mathrm{CDCl}_{3}, 125.76 \mathrm{MHz}\right): \delta 50.43,50.64$ (piperazine-C), 55.33 $\left(\mathrm{OCH}_{3}\right), 70.44(\mathrm{CH} 2), 111.05,118.28,120.94,123.17,123.68$, 128.32, 130.74, 130.95, 141.09, 152.23 (Ar \& thiophene-C), $155.42(\mathrm{C}=\mathrm{N}), 177.74(\mathrm{C}=\mathrm{S})$.

\section{Refinement}

Crystal data, data collection and structure refinement details are summarized in Table 2. The $\mathrm{C}$-bound $\mathrm{H}$ atoms were positioned geometrically and treated as riding atoms: $\mathrm{C}-\mathrm{H}$ $0.95-0.97 \AA$ with $\mathrm{U}_{\text {iso }}(\mathrm{H})=1.5 U_{\text {eq }}(\mathrm{C}$-methyl $)$ and $1.2 U_{\text {eq }}(\mathrm{C})$ for other $\mathrm{H}$ atoms. The thienyl ring is disordered over two positions and in the final refinement cycles, the occupancy of atoms $S 2 A$ and $\mathrm{C} 16 A$, and $\mathrm{S} 2 B$ and $\mathrm{C} 16 B$, were each fixed at 0.5 .

\section{Acknowledgements}

The authors extend their sincere appreciation to the Deanship of Scientific Research at King Saud University for funding this study through the Research Group Project No. PRG-1436-23. We also acknowledge financial support from the Spanish Ministerio de Economía y Competitividad (MINECO-13MAT2013-40950-R, FPI grant BES-2011-046948 to MSMA).

\section{References}

Agilent (2014). CrysAlis PRO. Agilent Technologies, Yarnton, England.

Al-Omar, M. A. (2010). Molecules, 15, 502-514.

Al-Omary, F. A. M., El-Emam, A. A., Ghabbour, H. A., Chidan Kumar, C. S., Quah, C. K. \& Fun, H.-K. (2015). Acta Cryst. E71, o175-o176.

Bansal, S., Bala, M., Suthar, S. K., Choudhary, S., Bhattacharya, S., Bhardwaj, V., Singla, S. \& Joseph, A. (2014). Eur. J. Med. Chem. 80, 167-174.

Du, Q.-R., Li, D.-D., Pi, Y.-Z., Li, J.-R., Sun, J., Fang, F., Zhong, W.-Q., Gong, H.-B. \& Zhu, H.-L. (2013). Eur. J. Med. Chem. 21, 22862297.

El-Emam, A. A., Al-Deeb, O. A., Al-Omar, M. A. \& Lehmann, J. (2004). Bioorg. Med. Chem. 12, 5107-5113.

El-Emam, A. A., Al-Omar, M. A., Al-Obaid, A.-R. M., Ng, S. W. \& Tiekink, E. R. T. (2013). Acta Cryst. E69, o684.

El-Emam, A. A., El-Brollosy, N. R., Attia, M. I., Said-Abdelbaky, M. \& García-Granda, S. (2012). Acta Cryst. E68, o2172-o2173.

Farrugia, L. J. (2012). J. Appl. Cryst. 45, 849-854.

Gamal El-Din, M. M., El-Gamal, M. I., Abdel-Maksoud, M. S., Yoo, K. H. \& Oh, C.-H. (2015). Eur. J. Med. Chem. 90, 45-52.

Groom, C. R. \& Allen, F. H. (2014). Angew. Chem. Int. Ed. 53, 662671.

Kadi, A. A., El-Brollosy, N. R., Al-Deeb, O. A., Habib, E. E., Ibrahim, T. M. \& El-Emam, A. A. (2007). Eur. J. Med. Chem. 42, 235242.

Li, P., Shi, L., Yang, X., Yang, L., Chen, X.-W., Wu, F., Shi, Q.-C., Xu, W.-M., He, M., Hu, D.-Y. \& Song, B.-A. (2014). Bioorg. Med. Chem. Lett. 24, 1677-1680.

Ma, L., Xiao, Y., Li, C., Xie, Z.-L., Li, Z.-L., Wang, Y.-T., Ma, H.-T., Zhu, H.-L., Wang, M.-H. \& Ye, Y.-H. (2013). Bioorg. Med. Chem. 21, 6763-6770.

Macrae, C. F., Bruno, I. J., Chisholm, J. A., Edgington, P. R., McCabe, P., Pidcock, E., Rodriguez-Monge, L., Taylor, R., van de Streek, J. \& Wood, P. A. (2008). J. Appl. Cryst. 41, 466-470.

Milinkevich, K. A., Yoo, C. L., Sparks, T. C., Lorsbach, B. A. \& Kurth, M. J. (2009). Bioorg. Med. Chem. Lett. 19, 5796-5798.

Ogata, M., Atobe, H., Kushida, H. \& Yamamoto, K. (1971). J. Antibiot. 24, 443-451.

Oxford Diffraction (2006). CrysAlis CCD and CrysAlis RED. Oxford Diffraction Ltd, Abingdon, England.

Pinna, G. A., Murineddu, G., Murruzzu, C., Zuco, V., Zunino, F., Cappelletti, G., Artali, R., Cignarella, G., Solano, L. \& Villa, S. (2009). ChemMedChem, 4, 998-1009.

Rane, R. A., Gutte, S. W. \& Sahu, N. U. (2012). Bioorg. Med. Chem. Lett. 22, 6429-6432.

Schlecker, R. \& Thieme, P. C. (1988). Tetrahedron, 44, 3289-3294.

Sheldrick, G. M. (2008). Acta Cryst. A64, 112-122.

Shi, W., Qian, X., Zhang, R. \& Song, G. (2001). J. Agric. Food Chem. 49, 124-130.

Summa, V., Petrocchi, A., Bonelli, F., Crescenzi, B., Donghi, M., Ferrara, M., Fiore, F., Gardelli, C., Gonzalez Paz, O., Hazuda, D. J., Jones, P., Kinzel, O., Laufer, R., Monteagudo, E., Muraglia, E., Nizi, E., Orvieto, F., Pace, P., Pescatore, G., Scarpelli, R., Stillmock, K., Witmer, M. V. \& Rowley, M. (2008). J. Med. Chem. 51, 5843-5855. 
Vardan, S., Smulyan, H., Mookherjee, S. \& Eich, R. (1983). Clin. Pharmacol. Ther. 34, 290-296.

Westrip, S. P. (2010). J. Appl. Cryst. 43, 920-925.

Wu, W., Chen, Q., Tai, A., Jiang, G. \& Ouyang, G. (2015). Bioorg. Med. Chem. Lett. 25, 2243-2246.
Zhang, K., Wang, P., Xuan, L.-N., Fu, X.-Y., Jing, F., Li, S., Liu, Y.-M. \& Chen, B.-Q. (2014). Bioorg. Med. Chem. Lett. 24, 5154-5156.

Zhang, Y., Zuniga, C., Kim, S.-J., Cai, D., Barlow, S., Salman, S., Coropceanu, V., Brédas, J.-L., Kippelen, B. \& Marder, S. (2011). Chem. Mater. 23, 4002-4015. 


\section{supporting information}

Acta Cryst. (2016). E72, 269-272 [doi:10.1107/S2056989016000992]

\section{Crystal structure of 3-\{[4-(2-methoxyphenyl) piperazin-1-yl]methyl\}-5-(thio-} phen-2-yl)-1,3,4-oxadiazole-2(3H)-thione

Monirah A. Al-Alshaikh, Hatem A. Abuelizz, Ali A. El-Emam, Mohammed S. M. Abdelbaky and Santiago Garcia-Granda

Computing details

Data collection: CrysAlis CCD (Oxford Diffraction, 2006); cell refinement: CrysAlis CCD (Oxford Diffraction, 2006); data reduction: CrysAlis RED (Oxford Diffraction, 2006); program(s) used to solve structure: SHELXS97 (Sheldrick, 2008); program(s) used to refine structure: SHELXL97 (Sheldrick, 2008); molecular graphics: ORTEP-3 for Windows (Farrugia, 2012) and Mercury (Macrae et al., 2008); software used to prepare material for publication: WinGX (Farrugia, 2012) and publCIF (Westrip, 2010).

3-\{[4-(2-Methoxyphenyl) piperazin-1-yl]methyl\}-5-(thiophen-2-yl)-1,3,4-oxadiazole-2(3H)-thione

Crystal data

$\mathrm{C}_{18} \mathrm{H}_{20} \mathrm{~N}_{4} \mathrm{O}_{2} \mathrm{~S}_{2}$

$M_{r}=388.5$

Monoclinic, $P 2_{1} / c$

Hall symbol: $-\mathrm{P} 2 \mathrm{ybc}$

$a=15.2925(2) \AA$

$b=10.0745(1) \AA$

$c=11.9726(1) \AA$

$\beta=93.413(1)^{\circ}$

$V=1841.28(3) \AA^{3}$

$Z=4$

\section{Data collection}

Agilent Xcalibur Ruby Gemini diffractometer

Radiation source: Enhance $(\mathrm{Cu}) \mathrm{X}$-ray Source Graphite monochromator

Detector resolution: 10.2673 pixels $\mathrm{mm}^{-1}$

$\omega$ scans

Absorption correction: multi-scan

(CrysAlis PRO; Agilent, 2014)

$T_{\min }=0.225, T_{\max }=0.315$

Refinement

Refinement on $F^{2}$

Least-squares matrix: full

$R\left[F^{2}>2 \sigma\left(F^{2}\right)\right]=0.045$

$w R\left(F^{2}\right)=0.113$

$S=1.04$
$F(000)=816$

$D_{\mathrm{x}}=1.401 \mathrm{Mg} \mathrm{m}^{-3}$

$\mathrm{Cu} K \alpha$ radiation, $\lambda=1.54184 \AA$

Cell parameters from 11296 reflections

$\theta=3.7-70.5^{\circ}$

$\mu=2.80 \mathrm{~mm}^{-1}$

$T=100 \mathrm{~K}$

Prism, colourless

$0.70 \times 0.51 \times 0.41 \mathrm{~mm}$

13494 measured reflections

3545 independent reflections

3401 reflections with $I>2 \sigma(I)$

$R_{\text {int }}=0.026$

$\theta_{\text {max }}=70.7^{\circ}, \theta_{\text {min }}=5.3^{\circ}$

$h=-18 \rightarrow 17$

$k=-8 \rightarrow 12$

$l=-14 \rightarrow 14$

3494 reflections

230 parameters

0 restraints

0 constraints 
Primary atom site location: structure-invariant direct methods

Secondary atom site location: difference Fourier map

Hydrogen site location: inferred from neighbouring sites
$\mathrm{H}$-atom parameters constrained

$$
\begin{aligned}
& w=1 /\left[\sigma^{2}\left(F_{\mathrm{o}}^{2}\right)+(0.0574 P)^{2}+2.048 P\right] \\
& \text { where } P=\left(F_{\mathrm{o}}^{2}+2 F_{\mathrm{c}}^{2}\right) / 3 \\
& (\Delta / \sigma)_{\max }<0.001 \\
& \Delta \rho_{\max }=0.95 \mathrm{e} \AA^{-3} \\
& \Delta \rho_{\min }=-0.65 \mathrm{e} \AA^{-3}
\end{aligned}
$$

\section{Special details}

Geometry. All e.s.d.'s (except the e.s.d. in the dihedral angle between two 1.s. planes) are estimated using the full covariance matrix. The cell e.s.d.'s are taken into account individually in the estimation of e.s.d.'s in distances, angles and torsion angles; correlations between e.s.d.'s in cell parameters are only used when they are defined by crystal symmetry. An approximate (isotropic) treatment of cell e.s.d.'s is used for estimating e.s.d.'s involving l.s. planes.

Refinement. Refinement of $F^{2}$ against ALL reflections. The weighted $R$-factor $w R$ and goodness of fit $S$ are based on $F^{2}$,

\begin{tabular}{|c|c|c|c|c|c|}
\hline & $x$ & $y$ & $z$ & $U_{\text {iso }} * / U_{\text {eq }}$ & Occ. $(<1)$ \\
\hline $\mathrm{S} 1$ & $0.89998(3)$ & $0.09896(5)$ & $0.99378(4)$ & $0.02414(15)$ & \\
\hline $\mathrm{S} 2 \mathrm{~A}$ & $0.87403(4)$ & $0.74266(6)$ & $1.04820(5)$ & $0.02497(16)$ & $0.7913(14)$ \\
\hline C16A & $0.94021(8)$ & $0.57554(13)$ & $1.21094(10)$ & $0.02497(16)$ & $0.7913(14)$ \\
\hline H16A & 0.9598 & 0.501 & 1.2508 & $0.03 *$ & $0.7913(14)$ \\
\hline S2B & $0.94021(8)$ & $0.57554(13)$ & $1.21094(10)$ & $0.02497(16)$ & $0.2087(14)$ \\
\hline $\mathrm{C} 16 \mathrm{~B}$ & $0.87403(4)$ & $0.74266(6)$ & $1.04820(5)$ & $0.02497(16)$ & $0.2087(14)$ \\
\hline H16B & 0.8502 & 0.779 & 0.9817 & $0.03^{*}$ & $0.2087(14)$ \\
\hline $\mathrm{O} 1$ & $0.90631(8)$ & $0.35048(13)$ & $1.06190(11)$ & 0.0189 & \\
\hline $\mathrm{O} 2$ & $0.43793(9)$ & $0.38449(17)$ & $0.90935(12)$ & $0.0312(4)$ & \\
\hline N3 & $0.85646(10)$ & $0.33316(16)$ & $0.89001(13)$ & $0.0192(3)$ & \\
\hline N1 & $0.55319(10)$ & $0.37224(16)$ & $0.74811(13)$ & 0.0198 & \\
\hline N4 & $0.85770(10)$ & $0.46847(16)$ & 0.91387 (13) & 0.0204 & \\
\hline N2 & $0.73225(10)$ & $0.29806(17)$ & $0.75238(13)$ & 0.0210 & \\
\hline $\mathrm{C} 1$ & $0.46191(12)$ & $0.37565(18)$ & $0.71654(16)$ & $0.0202(4)$ & \\
\hline $\mathrm{C} 15$ & $0.90176(11)$ & $0.58606(19)$ & $1.08927(15)$ & $0.0183(4)$ & \\
\hline $\mathrm{C} 14$ & $0.88737(11)$ & 0.47285 (19) & $1.01696(15)$ & $0.0180(4)$ & \\
\hline $\mathrm{C} 13$ & $0.88567(12)$ & $0.26006(19)$ & $0.97769(15)$ & $0.0187(4)$ & \\
\hline C6 & $0.40227(13)$ & $0.3813(2)$ & $0.80197(17)$ & $0.0229(4)$ & \\
\hline C9 & $0.67626(13)$ & $0.2461(2)$ & $0.83683(17)$ & $0.0237(4)$ & \\
\hline H9A & 0.6806 & 0.3025 & 0.9026 & $0.028 *$ & \\
\hline H9B & 0.6955 & 0.1577 & 0.8589 & $0.028 *$ & \\
\hline C11 & $0.61058(12)$ & $0.4202(2)$ & $0.66366(16)$ & $0.0231(4)$ & \\
\hline H11A & 0.5908 & 0.5066 & 0.6368 & $0.028^{*}$ & \\
\hline H11B & 0.6088 & 0.3596 & 0.6007 & $0.028^{*}$ & \\
\hline $\mathrm{C} 4$ & $0.28120(13)$ & $0.3853(2)$ & $0.66375(19)$ & $0.0278(5)$ & \\
\hline $\mathrm{H} 4$ & 0.2211 & 0.3875 & 0.6463 & $0.033^{*}$ & \\
\hline $\mathrm{C} 3$ & $0.33874(14)$ & $0.3826(2)$ & $0.57913(18)$ & $0.0284(5)$ & \\
\hline $\mathrm{H} 3$ & 0.3177 & 0.3844 & 0.5046 & $0.034 *$ & \\
\hline $\mathrm{C} 2$ & $0.42871(13)$ & $0.3770(2)$ & $0.60627(17)$ & $0.0249(4)$ & \\
\hline $\mathrm{H} 2$ & 0.4672 & 0.3741 & 0.5491 & $0.03 *$ & \\
\hline
\end{tabular}
conventional $R$-factors $R$ are based on $F$, with $F$ set to zero for negative $F^{2}$. The threshold expression of $F^{2}>\sigma\left(F^{2}\right)$ is used only for calculating $R$-factors $(\mathrm{gt})$ etc. and is not relevant to the choice of reflections for refinement. $R$-factors based on $F^{2}$ are statistically about twice as large as those based on $F$, and $R$ - factors based on ALL data will be even larger.

Fractional atomic coordinates and isotropic or equivalent isotropic displacement parameters $\left(\AA^{2}\right)$ 


$\begin{array}{lllll}\text { C10 } & 0.70379(12) & 0.4304(2) & 0.71497(16) & 0.0228(4) \\ \text { H10A } & 0.7424 & 0.4638 & 0.66 & 0.027^{*} \\ \text { H10B } & 0.7058 & 0.4912 & 0.7779 & 0.027^{*} \\ \text { C8 } & 0.58162(13) & 0.2411(2) & 0.78978(18) & 0.0240(4) \\ \text { H8A } & 0.5763 & 0.177 & 0.7292 & 0.029^{*} \\ \text { H8B } & 0.5441 & 0.2125 & 0.8478 & 0.029^{*} \\ \text { C5 } & 0.31283(13) & 0.3849(2) & 0.77469(18) & 0.0275(5) \\ \text { H5 } & 0.2738 & 0.3869 & 0.8313 & 0.033^{*} \\ \text { C12 } & 0.82390(12) & 0.2821(2) & 0.77866(15) & 0.0224(4) \\ \text { H12A } & 0.8555 & 0.3268 & 0.7217 & 0.027^{*} \\ \text { H12B } & 0.8379 & 0.1883 & 0.7749 & 0.027^{*} \\ \text { C17 } & 0.93757(13) & 0.7167(2) & 1.24746(17) & 0.0268(4) \\ \text { H17 } & 0.9571 & 0.7421 & 1.3193 & 0.032^{*} \\ \text { C7 } & 0.38537(15) & 0.4430(3) & 0.99152(19) & 0.0342(5) \\ \text { H7A } & 0.4167 & 0.4403 & 1.0634 & 0.051^{*} \\ \text { H7B } & 0.3316 & 0.3943 & 0.9947 & 0.051^{*} \\ \text { H7C } & 0.3726 & 0.5335 & 0.9715 & 0.051^{*} \\ \text { C18 } & 0.90558(13) & 0.8060(2) & 1.1716(2) & 0.0297(5) \\ \text { H18 } & 0.9016 & 0.8961 & 1.1874 & 0.036^{*}\end{array}$

Atomic displacement parameters $\left(\AA^{2}\right)$

\begin{tabular}{lllllll}
\hline & $U^{11}$ & $U^{22}$ & $U^{33}$ & $U^{12}$ & $U^{13}$ & $U^{23}$ \\
\hline S1 & $0.0282(3)$ & $0.0197(3)$ & $0.0246(3)$ & $0.00178(18)$ & $0.00181(19)$ & $0.00051(18)$ \\
S2A & $0.0229(3)$ & $0.0257(3)$ & $0.0265(3)$ & $-0.0011(2)$ & $0.0022(2)$ & $-0.0008(2)$ \\
C16A & $0.0229(3)$ & $0.0257(3)$ & $0.0265(3)$ & $-0.0011(2)$ & $0.0022(2)$ & $-0.0008(2)$ \\
S2B & $0.0229(3)$ & $0.0257(3)$ & $0.0265(3)$ & $-0.0011(2)$ & $0.0022(2)$ & $-0.0008(2)$ \\
C16B & $0.0229(3)$ & $0.0257(3)$ & $0.0265(3)$ & $-0.0011(2)$ & $0.0022(2)$ & $-0.0008(2)$ \\
O1 & $0.0189(6)$ & $0.0199(6)$ & $0.0177(6)$ & $0.0015(5)$ & $0.0001(5)$ & $0.0003(5)$ \\
O2 & $0.0213(7)$ & $0.0510(10)$ & $0.0218(7)$ & $-0.0010(6)$ & $0.0057(6)$ & $-0.0044(6)$ \\
N3 & $0.0175(7)$ & $0.0216(8)$ & $0.0184(8)$ & $0.0028(6)$ & $0.0006(6)$ & $-0.0007(6)$ \\
N1 & $0.0161(8)$ & $0.0234(8)$ & $0.0201(8)$ & $0.0005(6)$ & $0.0037(6)$ & $0.0027(6)$ \\
N4 & $0.0196(8)$ & $0.0219(8)$ & $0.0197(8)$ & $0.0030(6)$ & $0.0022(6)$ & $0.0002(6)$ \\
N2 & $0.0170(8)$ & $0.0260(8)$ & $0.0201(8)$ & $0.0035(6)$ & $0.0011(6)$ & $0.0002(7)$ \\
C1 & $0.0175(9)$ & $0.0190(9)$ & $0.0244(10)$ & $-0.0012(7)$ & $0.0030(7)$ & $0.0003(7)$ \\
C15 & $0.0141(8)$ & $0.0219(9)$ & $0.0191(9)$ & $0.0009(7)$ & $0.0019(7)$ & $0.0014(7)$ \\
C14 & $0.0128(8)$ & $0.0209(9)$ & $0.0204(9)$ & $0.0022(7)$ & $0.0029(7)$ & $0.0023(7)$ \\
C13 & $0.0143(8)$ & $0.0241(10)$ & $0.0182(9)$ & $0.0006(7)$ & $0.0031(7)$ & $-0.0016(7)$ \\
C6 & $0.0216(10)$ & $0.0233(10)$ & $0.0239(10)$ & $-0.0022(8)$ & $0.0034(8)$ & $0.0000(8)$ \\
C9 & $0.0218(10)$ & $0.0248(10)$ & $0.0245(10)$ & $0.0031(8)$ & $0.0021(8)$ & $0.0047(8)$ \\
C11 & $0.0177(9)$ & $0.0309(11)$ & $0.0211(9)$ & $0.0008(8)$ & $0.0031(7)$ & $0.0060(8)$ \\
C4 & $0.0163(9)$ & $0.0309(11)$ & $0.0360(11)$ & $-0.0025(8)$ & $0.0000(8)$ & $0.0014(9)$ \\
C3 & $0.0228(10)$ & $0.0351(12)$ & $0.0267(10)$ & $-0.0024(8)$ & $-0.0019(8)$ & $0.0032(9)$ \\
C2 & $0.0197(9)$ & $0.0309(11)$ & $0.0246(10)$ & $-0.0008(8)$ & $0.0042(8)$ & $0.0010(8)$ \\
C10 & $0.0177(9)$ & $0.0284(10)$ & $0.0224(9)$ & $-0.0002(8)$ & $0.0024(7)$ & $0.0055(8)$ \\
C8 & $0.0203(9)$ & $0.0228(10)$ & $0.0290(10)$ & $-0.0003(7)$ & $0.0030(8)$ & $0.0035(8)$ \\
C5 & $0.0197(10)$ & $0.0331(11)$ & $0.0307(11)$ & $-0.0032(8)$ & $0.0087(8)$ & $-0.0001(9)$ \\
C12 & $0.0204(9)$ & $0.0295(10)$ & $0.0174(9)$ & $0.0047(8)$ & $0.0017(7)$ & $-0.0042(8)$ \\
& & & & & &
\end{tabular}




\begin{tabular}{lllllll}
$\mathrm{C} 17$ & $0.0173(9)$ & $0.0420(12)$ & $0.0211(9)$ & $-0.0069(8)$ & $0.0027(7)$ & $-0.0057(9)$ \\
$\mathrm{C} 7$ & $0.0326(11)$ & $0.0442(13)$ & $0.0272(11)$ & $-0.0067(10)$ & $0.0117(9)$ & $-0.0083(10)$ \\
$\mathrm{C} 18$ & $0.0240(10)$ & $0.0230(10)$ & $0.0432(12)$ & $-0.0017(8)$ & $0.0102(9)$ & $-0.0017(9)$ \\
\hline
\end{tabular}

Geometric parameters $\left(A,{ }^{\circ}\right)$

\begin{tabular}{|c|c|c|c|}
\hline $\mathrm{S} 1-\mathrm{C} 13$ & $1.647(2)$ & C9-H9A & 0.97 \\
\hline $\mathrm{S} 2 \mathrm{~A}-\mathrm{C} 18$ & $1.655(2)$ & C9-H9B & 0.97 \\
\hline $\mathrm{S} 2 \mathrm{~A}-\mathrm{C} 15$ & $1.6988(19)$ & $\mathrm{C} 11-\mathrm{C} 10$ & $1.522(3)$ \\
\hline $\mathrm{C} 16 \mathrm{~A}-\mathrm{C} 17$ & $1.489(3)$ & $\mathrm{C} 11-\mathrm{H} 11 \mathrm{~A}$ & 0.97 \\
\hline $\mathrm{C} 16 \mathrm{~A}-\mathrm{C} 15$ & $1.542(2)$ & $\mathrm{C} 11-\mathrm{H} 11 \mathrm{~B}$ & 0.97 \\
\hline $\mathrm{C} 16 \mathrm{~A}-\mathrm{H} 16 \mathrm{~A}$ & 0.93 & $\mathrm{C} 4-\mathrm{C} 3$ & $1.381(3)$ \\
\hline $\mathrm{O} 1-\mathrm{C} 14$ & $1.369(2)$ & $\mathrm{C} 4-\mathrm{C} 5$ & $1.386(3)$ \\
\hline $\mathrm{O} 1-\mathrm{C} 13$ & $1.381(2)$ & $\mathrm{C} 4-\mathrm{H} 4$ & 0.93 \\
\hline $\mathrm{O} 2-\mathrm{C} 6$ & $1.367(3)$ & $\mathrm{C} 3-\mathrm{C} 2$ & $1.396(3)$ \\
\hline $\mathrm{O} 2-\mathrm{C} 7$ & $1.434(3)$ & $\mathrm{C} 3-\mathrm{H} 3$ & 0.93 \\
\hline $\mathrm{N} 3-\mathrm{C} 13$ & $1.337(2)$ & $\mathrm{C} 2-\mathrm{H} 2$ & 0.93 \\
\hline N3-N4 & $1.393(2)$ & $\mathrm{C} 10-\mathrm{H} 10 \mathrm{~A}$ & 0.97 \\
\hline $\mathrm{N} 3-\mathrm{C} 12$ & $1.487(2)$ & $\mathrm{C} 10-\mathrm{H} 10 \mathrm{~B}$ & 0.97 \\
\hline $\mathrm{N} 1-\mathrm{C} 1$ & $1.425(2)$ & $\mathrm{C} 8-\mathrm{H} 8 \mathrm{~A}$ & 0.97 \\
\hline $\mathrm{N} 1-\mathrm{C} 11$ & $1.460(2)$ & $\mathrm{C} 8-\mathrm{H} 8 \mathrm{~B}$ & 0.97 \\
\hline $\mathrm{N} 1-\mathrm{C} 8$ & $1.469(2)$ & $\mathrm{C} 5-\mathrm{H} 5$ & 0.93 \\
\hline $\mathrm{N} 4-\mathrm{C} 14$ & $1.290(2)$ & $\mathrm{C} 12-\mathrm{H} 12 \mathrm{~A}$ & 0.97 \\
\hline $\mathrm{N} 2-\mathrm{C} 12$ & $1.427(2)$ & $\mathrm{C} 12-\mathrm{H} 12 \mathrm{~B}$ & 0.97 \\
\hline N2-C9 & $1.460(2)$ & $\mathrm{C} 17-\mathrm{C} 18$ & $1.349(3)$ \\
\hline $\mathrm{N} 2-\mathrm{C} 10$ & $1.464(3)$ & C17-H17 & 0.93 \\
\hline $\mathrm{C} 1-\mathrm{C} 2$ & $1.386(3)$ & $\mathrm{C} 7-\mathrm{H} 7 \mathrm{~A}$ & 0.96 \\
\hline $\mathrm{C} 1-\mathrm{C} 6$ & $1.412(3)$ & $\mathrm{C} 7-\mathrm{H} 7 \mathrm{~B}$ & 0.96 \\
\hline $\mathrm{C} 15-\mathrm{C} 14$ & $1.441(3)$ & $\mathrm{C} 7-\mathrm{H} 7 \mathrm{C}$ & 0.96 \\
\hline $\mathrm{C} 6-\mathrm{C} 5$ & $1.388(3)$ & $\mathrm{C} 18-\mathrm{H} 18$ & 0.93 \\
\hline $\mathrm{C} 9-\mathrm{C} 8$ & $1.522(3)$ & & \\
\hline $\mathrm{C} 18-\mathrm{S} 2 \mathrm{~A}-\mathrm{C} 15$ & $92.58(10)$ & $\mathrm{C} 3-\mathrm{C} 4-\mathrm{C} 5$ & $120.10(19)$ \\
\hline $\mathrm{C} 17-\mathrm{C} 16 \mathrm{~A}-\mathrm{C} 15$ & $101.32(13)$ & $\mathrm{C} 3-\mathrm{C} 4-\mathrm{H} 4$ & 120 \\
\hline $\mathrm{C} 17-\mathrm{C} 16 \mathrm{~A}-\mathrm{H} 16 \mathrm{~A}$ & 129.3 & $\mathrm{C} 5-\mathrm{C} 4-\mathrm{H} 4$ & 120 \\
\hline $\mathrm{C} 15-\mathrm{C} 16 \mathrm{~A}-\mathrm{H} 16 \mathrm{~A}$ & 129.3 & $\mathrm{C} 4-\mathrm{C} 3-\mathrm{C} 2$ & $119.49(19)$ \\
\hline $\mathrm{C} 14-\mathrm{O} 1-\mathrm{C} 13$ & $105.85(14)$ & $\mathrm{C} 4-\mathrm{C} 3-\mathrm{H} 3$ & 120.3 \\
\hline $\mathrm{C} 6-\mathrm{O} 2-\mathrm{C} 7$ & $116.52(17)$ & $\mathrm{C} 2-\mathrm{C} 3-\mathrm{H} 3$ & 120.3 \\
\hline $\mathrm{C} 13-\mathrm{N} 3-\mathrm{N} 4$ & $112.23(15)$ & $\mathrm{C} 1-\mathrm{C} 2-\mathrm{C} 3$ & $121.48(19)$ \\
\hline $\mathrm{C} 13-\mathrm{N} 3-\mathrm{C} 12$ & $126.27(17)$ & $\mathrm{C} 1-\mathrm{C} 2-\mathrm{H} 2$ & 119.3 \\
\hline $\mathrm{N} 4-\mathrm{N} 3-\mathrm{C} 12$ & $121.49(15)$ & $\mathrm{C} 3-\mathrm{C} 2-\mathrm{H} 2$ & 119.3 \\
\hline $\mathrm{C} 1-\mathrm{N} 1-\mathrm{C} 11$ & $115.34(15)$ & $\mathrm{N} 2-\mathrm{C} 10-\mathrm{C} 11$ & $108.46(16)$ \\
\hline $\mathrm{C} 1-\mathrm{N} 1-\mathrm{C} 8$ & $112.16(15)$ & $\mathrm{N} 2-\mathrm{C} 10-\mathrm{H} 10 \mathrm{~A}$ & 110 \\
\hline $\mathrm{C} 11-\mathrm{N} 1-\mathrm{C} 8$ & $110.80(15)$ & $\mathrm{C} 11-\mathrm{C} 10-\mathrm{H} 10 \mathrm{~A}$ & 110 \\
\hline $\mathrm{C} 14-\mathrm{N} 4-\mathrm{N} 3$ & $103.26(15)$ & $\mathrm{N} 2-\mathrm{C} 10-\mathrm{H} 10 \mathrm{~B}$ & 110 \\
\hline $\mathrm{C} 12-\mathrm{N} 2-\mathrm{C} 9$ & $114.55(15)$ & $\mathrm{C} 11-\mathrm{C} 10-\mathrm{H} 10 \mathrm{~B}$ & 110 \\
\hline $\mathrm{C} 12-\mathrm{N} 2-\mathrm{C} 10$ & $116.12(16)$ & $\mathrm{H} 10 \mathrm{~A}-\mathrm{C} 10-\mathrm{H} 10 \mathrm{~B}$ & 108.4 \\
\hline $\mathrm{C} 9-\mathrm{N} 2-\mathrm{C} 10$ & $111.25(15)$ & $\mathrm{N} 1-\mathrm{C} 8-\mathrm{C} 9$ & $110.57(16)$ \\
\hline
\end{tabular}




\begin{tabular}{|c|c|c|c|}
\hline $\mathrm{C} 2-\mathrm{C} 1-\mathrm{C} 6$ & $118.29(18)$ & $\mathrm{N} 1-\mathrm{C} 8-\mathrm{H} 8 \mathrm{~A}$ & 109.5 \\
\hline $\mathrm{C} 2-\mathrm{C} 1-\mathrm{N} 1$ & $123.40(17)$ & $\mathrm{C} 9-\mathrm{C} 8-\mathrm{H} 8 \mathrm{~A}$ & 109.5 \\
\hline $\mathrm{C} 6-\mathrm{C} 1-\mathrm{N} 1$ & $118.28(17)$ & $\mathrm{N} 1-\mathrm{C} 8-\mathrm{H} 8 \mathrm{~B}$ & 109.5 \\
\hline $\mathrm{C} 14-\mathrm{C} 15-\mathrm{C} 16 \mathrm{~A}$ & $123.32(15)$ & $\mathrm{C} 9-\mathrm{C} 8-\mathrm{H} 8 \mathrm{~B}$ & 109.5 \\
\hline $\mathrm{C} 14-\mathrm{C} 15-\mathrm{S} 2 \mathrm{~A}$ & $122.32(14)$ & $\mathrm{H} 8 \mathrm{~A}-\mathrm{C} 8-\mathrm{H} 8 \mathrm{~B}$ & 108.1 \\
\hline $\mathrm{C} 16 \mathrm{~A}-\mathrm{C} 15-\mathrm{S} 2 \mathrm{~A}$ & $114.33(12)$ & $\mathrm{C} 4-\mathrm{C} 5-\mathrm{C} 6$ & $120.56(19)$ \\
\hline $\mathrm{N} 4-\mathrm{C} 14-\mathrm{O} 1$ & $113.53(16)$ & $\mathrm{C} 4-\mathrm{C} 5-\mathrm{H} 5$ & 119.7 \\
\hline $\mathrm{N} 4-\mathrm{C} 14-\mathrm{C} 15$ & $129.37(18)$ & $\mathrm{C} 6-\mathrm{C} 5-\mathrm{H} 5$ & 119.7 \\
\hline $\mathrm{O} 1-\mathrm{C} 14-\mathrm{C} 15$ & $117.09(16)$ & $\mathrm{N} 2-\mathrm{C} 12-\mathrm{N} 3$ & $115.51(15)$ \\
\hline $\mathrm{N} 3-\mathrm{C} 13-\mathrm{O} 1$ & $105.12(16)$ & $\mathrm{N} 2-\mathrm{C} 12-\mathrm{H} 12 \mathrm{~A}$ & 108.4 \\
\hline $\mathrm{N} 3-\mathrm{C} 13-\mathrm{S} 1$ & $132.09(15)$ & $\mathrm{N} 3-\mathrm{C} 12-\mathrm{H} 12 \mathrm{~A}$ & 108.4 \\
\hline $\mathrm{O} 1-\mathrm{C} 13-\mathrm{S} 1$ & $122.77(14)$ & $\mathrm{N} 2-\mathrm{C} 12-\mathrm{H} 12 \mathrm{~B}$ & 108.4 \\
\hline $\mathrm{O} 2-\mathrm{C} 6-\mathrm{C} 5$ & $123.60(18)$ & $\mathrm{N} 3-\mathrm{C} 12-\mathrm{H} 12 \mathrm{~B}$ & 108.4 \\
\hline $\mathrm{O} 2-\mathrm{C} 6-\mathrm{C} 1$ & $116.35(17)$ & $\mathrm{H} 12 \mathrm{~A}-\mathrm{C} 12-\mathrm{H} 12 \mathrm{~B}$ & 107.5 \\
\hline $\mathrm{C} 5-\mathrm{C} 6-\mathrm{C} 1$ & $120.05(19)$ & $\mathrm{C} 18-\mathrm{C} 17-\mathrm{C} 16 \mathrm{~A}$ & $117.01(18)$ \\
\hline $\mathrm{N} 2-\mathrm{C} 9-\mathrm{C} 8$ & $109.84(16)$ & $\mathrm{C} 18-\mathrm{C} 17-\mathrm{H} 17$ & 121.5 \\
\hline $\mathrm{N} 2-\mathrm{C} 9-\mathrm{H} 9 \mathrm{~A}$ & 109.7 & $\mathrm{C} 16 \mathrm{~A}-\mathrm{C} 17-\mathrm{H} 17$ & 121.5 \\
\hline $\mathrm{C} 8-\mathrm{C} 9-\mathrm{H} 9 \mathrm{~A}$ & 109.7 & $\mathrm{O} 2-\mathrm{C} 7-\mathrm{H} 7 \mathrm{~A}$ & 109.5 \\
\hline $\mathrm{N} 2-\mathrm{C} 9-\mathrm{H} 9 \mathrm{~B}$ & 109.7 & $\mathrm{O} 2-\mathrm{C} 7-\mathrm{H} 7 \mathrm{~B}$ & 109.5 \\
\hline $\mathrm{C} 8-\mathrm{C} 9-\mathrm{H} 9 \mathrm{~B}$ & 109.7 & $\mathrm{H} 7 \mathrm{~A}-\mathrm{C} 7-\mathrm{H} 7 \mathrm{~B}$ & 109.5 \\
\hline $\mathrm{H} 9 \mathrm{~A}-\mathrm{C} 9-\mathrm{H} 9 \mathrm{~B}$ & 108.2 & $\mathrm{O} 2-\mathrm{C} 7-\mathrm{H} 7 \mathrm{C}$ & 109.5 \\
\hline $\mathrm{N} 1-\mathrm{C} 11-\mathrm{C} 10$ & $109.26(15)$ & $\mathrm{H} 7 \mathrm{~A}-\mathrm{C} 7-\mathrm{H} 7 \mathrm{C}$ & 109.5 \\
\hline $\mathrm{N} 1-\mathrm{C} 11-\mathrm{H} 11 \mathrm{~A}$ & 109.8 & $\mathrm{H} 7 \mathrm{~B}-\mathrm{C} 7-\mathrm{H} 7 \mathrm{C}$ & 109.5 \\
\hline $\mathrm{C} 10-\mathrm{C} 11-\mathrm{H} 11 \mathrm{~A}$ & 109.8 & $\mathrm{C} 17-\mathrm{C} 18-\mathrm{S} 2 \mathrm{~A}$ & $114.76(17)$ \\
\hline $\mathrm{N} 1-\mathrm{C} 11-\mathrm{H} 11 \mathrm{~B}$ & 109.8 & $\mathrm{C} 17-\mathrm{C} 18-\mathrm{H} 18$ & 122.6 \\
\hline $\mathrm{C} 10-\mathrm{C} 11-\mathrm{H} 11 \mathrm{~B}$ & 109.8 & $\mathrm{~S} 2 \mathrm{~A}-\mathrm{C} 18-\mathrm{H} 18$ & 122.6 \\
\hline $\mathrm{H} 11 \mathrm{~A}-\mathrm{C} 11-\mathrm{H} 11 \mathrm{~B}$ & 108.3 & & \\
\hline $\mathrm{C} 13-\mathrm{N} 3-\mathrm{N} 4-\mathrm{C} 14$ & $-0.5(2)$ & $\mathrm{N} 1-\mathrm{C} 1-\mathrm{C} 6-\mathrm{O} 2$ & $0.7(3)$ \\
\hline $\mathrm{C} 12-\mathrm{N} 3-\mathrm{N} 4-\mathrm{C} 14$ & $178.38(15)$ & $\mathrm{C} 2-\mathrm{C} 1-\mathrm{C} 6-\mathrm{C} 5$ & $1.5(3)$ \\
\hline $\mathrm{C} 11-\mathrm{N} 1-\mathrm{C} 1-\mathrm{C} 2$ & $22.5(3)$ & $\mathrm{N} 1-\mathrm{C} 1-\mathrm{C} 6-\mathrm{C} 5$ & $179.97(18)$ \\
\hline $\mathrm{C} 8-\mathrm{N} 1-\mathrm{C} 1-\mathrm{C} 2$ & $-105.7(2)$ & $\mathrm{C} 12-\mathrm{N} 2-\mathrm{C} 9-\mathrm{C} 8$ & $-167.82(16)$ \\
\hline $\mathrm{C} 11-\mathrm{N} 1-\mathrm{C} 1-\mathrm{C} 6$ & $-155.90(18)$ & $\mathrm{C} 10-\mathrm{N} 2-\mathrm{C} 9-\mathrm{C} 8$ & $58.0(2)$ \\
\hline $\mathrm{C} 8-\mathrm{N} 1-\mathrm{C} 1-\mathrm{C} 6$ & $76.0(2)$ & $\mathrm{C} 1-\mathrm{N} 1-\mathrm{C} 11-\mathrm{C} 10$ & $171.49(16)$ \\
\hline $\mathrm{C} 17-\mathrm{C} 16 \mathrm{~A}-\mathrm{C} 15-\mathrm{C} 14$ & $-177.76(16)$ & $\mathrm{C} 8-\mathrm{N} 1-\mathrm{C} 11-\mathrm{C} 10$ & $-59.7(2)$ \\
\hline $\mathrm{C} 17-\mathrm{C} 16 \mathrm{~A}-\mathrm{C} 15-\mathrm{S} 2 \mathrm{~A}$ & $0.16(15)$ & $\mathrm{C} 5-\mathrm{C} 4-\mathrm{C} 3-\mathrm{C} 2$ & $1.1(3)$ \\
\hline $\mathrm{C} 18-\mathrm{S} 2 \mathrm{~A}-\mathrm{C} 15-\mathrm{C} 14$ & $177.79(16)$ & $\mathrm{C} 6-\mathrm{C} 1-\mathrm{C} 2-\mathrm{C} 3$ & $-0.5(3)$ \\
\hline $\mathrm{C} 18-\mathrm{S} 2 \mathrm{~A}-\mathrm{C} 15-\mathrm{C} 16 \mathrm{~A}$ & $-0.16(13)$ & $\mathrm{N} 1-\mathrm{C} 1-\mathrm{C} 2-\mathrm{C} 3$ & $-178.91(19)$ \\
\hline $\mathrm{N} 3-\mathrm{N} 4-\mathrm{C} 14-\mathrm{O} 1$ & $0.53(19)$ & $\mathrm{C} 4-\mathrm{C} 3-\mathrm{C} 2-\mathrm{C} 1$ & $-0.8(3)$ \\
\hline $\mathrm{N} 3-\mathrm{N} 4-\mathrm{C} 14-\mathrm{C} 15$ & $-178.64(17)$ & $\mathrm{C} 12-\mathrm{N} 2-\mathrm{C} 10-\mathrm{C} 11$ & $165.92(15)$ \\
\hline $\mathrm{C} 13-\mathrm{O} 1-\mathrm{C} 14-\mathrm{N} 4$ & $-0.4(2)$ & $\mathrm{C} 9-\mathrm{N} 2-\mathrm{C} 10-\mathrm{C} 11$ & $-60.7(2)$ \\
\hline $\mathrm{C} 13-\mathrm{O} 1-\mathrm{C} 14-\mathrm{C} 15$ & $178.90(15)$ & $\mathrm{N} 1-\mathrm{C} 11-\mathrm{C} 10-\mathrm{N} 2$ & $60.7(2)$ \\
\hline $\mathrm{C} 16 \mathrm{~A}-\mathrm{C} 15-\mathrm{C} 14-\mathrm{N} 4$ & $-178.37(16)$ & $\mathrm{C} 1-\mathrm{N} 1-\mathrm{C} 8-\mathrm{C} 9$ & $-172.53(16)$ \\
\hline $\mathrm{S} 2 \mathrm{~A}-\mathrm{C} 15-\mathrm{C} 14-\mathrm{N} 4$ & $3.9(3)$ & $\mathrm{C} 11-\mathrm{N} 1-\mathrm{C} 8-\mathrm{C} 9$ & $57.0(2)$ \\
\hline $\mathrm{C} 16 \mathrm{~A}-\mathrm{C} 15-\mathrm{C} 14-\mathrm{O} 1$ & $2.5(2)$ & $\mathrm{N} 2-\mathrm{C} 9-\mathrm{C} 8-\mathrm{N} 1$ & $-55.2(2)$ \\
\hline $\mathrm{S} 2 \mathrm{~A}-\mathrm{C} 15-\mathrm{C} 14-\mathrm{O} 1$ & $-175.28(13)$ & $\mathrm{C} 3-\mathrm{C} 4-\mathrm{C} 5-\mathrm{C} 6$ & $-0.2(3)$ \\
\hline $\mathrm{N} 4-\mathrm{N} 3-\mathrm{C} 13-\mathrm{O} 1$ & $0.29(19)$ & $\mathrm{O} 2-\mathrm{C} 6-\mathrm{C} 5-\mathrm{C} 4$ & $178.1(2)$ \\
\hline $\mathrm{C} 12-\mathrm{N} 3-\mathrm{C} 13-\mathrm{O} 1$ & $-178.53(15)$ & $\mathrm{C} 1-\mathrm{C} 6-\mathrm{C} 5-\mathrm{C} 4$ & $-1.2(3)$ \\
\hline
\end{tabular}




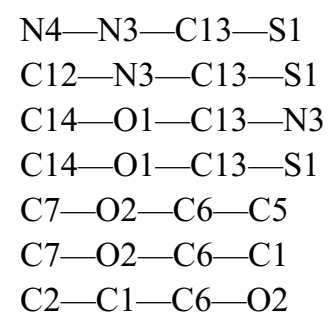

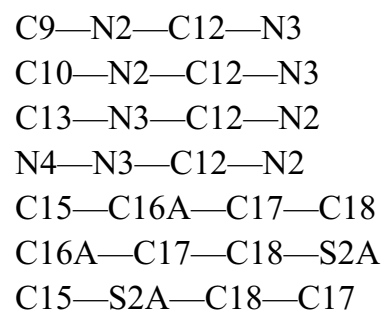

$-52.8(2)$

$79.1(2)$

$110.8(2)$

$-67.9(2)$

$-0.1(2)$

$0.0(2)$

$0.11(17)$

Hydrogen-bond geometry $\left(\AA,{ }^{\circ}\right)$

$\mathrm{Cg} 1$ is the centroid of the $\mathrm{S} 2 A / \mathrm{C} 15 / \mathrm{C} 16 A / \mathrm{C} 17 / \mathrm{C} 18$ ring.

\begin{tabular}{lllll}
\hline$D-\mathrm{H} \cdots A$ & $D-\mathrm{H}$ & $\mathrm{H} \cdots A$ & $D \cdots A$ & $D-\mathrm{H} \cdots A$ \\
\hline $\mathrm{C} 12-\mathrm{H} 12 A \cdots \mathrm{S} 1^{\mathrm{i}}$ & 0.97 & 2.95 & $3.860(2)$ & 157 \\
$\mathrm{C} 17-\mathrm{H} 17 \cdots \mathrm{O} 1^{\mathrm{ii}}$ & 0.93 & 2.69 & $3.475(2)$ & 143 \\
$\mathrm{C} 5-\mathrm{H} 5 \cdots C g 1^{1 i i}$ & 0.93 & 2.95 & $3.660(2)$ & 135
\end{tabular}

Symmetry codes: (i) $x,-y+1 / 2, z-1 / 2$; (ii) $-x+2, y+1 / 2,-z+5 / 2$; (iii) $-x+1,-y+1,-z+2$. 